\title{
The impact of distractions and interruptions in the operating room on patient safety and the operating room team: An integrative
} review

Follow this and additional works at: https://www.journal.acorn.org.au/jpn

Part of the Health Services Administration Commons, Health Services Research Commons, Perioperative, Operating Room and Surgical Nursing Commons, and the Surgery Commons

\section{(c) (1)}

This work is licensed under a Creative Commons Attribution 4.0 License.

\section{Recommended Citation}

Mackenzie, Sonia and Foran, Paula (2020) "The impact of distractions and interruptions in the operating room on patient safety and the operating room team: An integrative review," Journal of Perioperative Nursing: Vol. 33 : Iss. 3 , Article 6.

Available at: https://doi.org/10.26550/2209-1092.1098

https://www.journal.acorn.org.au/jpn/vol33/iss3/6

This Article is brought to you for free and open access by Journal of Perioperative Nursing. It has been accepted for inclusion in Journal of Perioperative Nursing by an authorized editor of Journal of Perioperative Nursing. 


\section{Authors}

Sonia Mackenzie

MCN (Perioperative), Grad Dip

(Perioperative), RN

Paula Foran

PhD, MACN, FACORN

\section{The impact of distractions and interruptions in the operating room on patient safety and the operating room team: An integrative review}

\begin{abstract}
Problem identification: In the operating room (OR), distractions and interruptions are frequent, impacting patient safety, coordination and efficiency and causing errors and patient harm. The OR team is impacted while attempting to perform critical work. This review explores the impact of distractions and interruptions in the OR on patient safety and the OR team.
\end{abstract}

Literature search: Inclusion and exclusion criteria were determined. Six databases were searched with the search criteria for inclusion being in English, peer-reviewed and published between 2014 and 2019. In total 296 papers were identified.

Data evaluation synthesis: Duplicates were removed, and 195 papers were screened using inclusion and exclusion criteria. Fourteen studies were included in the review: 12 were quantitative reviews and two were mixedmethod reviews. Methodological quality was assessed using the mixed methods appraisal tool (MMAT), with scores between 60 and 90 per cent. A thematic analysis revealed observational study themes of types, frequency and severity of distractions and interruptions, and impacts upon mental workload, patient safety and the OR team. Simulation study themes included types of distractions and interruptions, and impact on mental workload, clinical decision-making, surgical performance and nurses.

Implications for practice or research: The heterogeneity of the literature and paucity of recent nursing and anaesthetic studies highlights that further research is necessary. Nurses can educate and develop policies and interventions to reduce distractions, enhancing patient safety and decreasing the negative impact upon their colleagues and teams.

Keywords: distractions, interruptions, disruptions, operating room, perioperative, patient safety. 


\section{Problem identification}

Operating rooms (ORs) are complex environments in which the whole OR team (surgical, anaesthetic and nursing personnel) experiences high levels of cognitive demand while maintaining concentration and performing often difficult and highly precise tasks ${ }^{1-3}$. In the OR, distractions and interruptions are ubiquitous and varied yet there remains a paucity of empirical literature on the specific effects they have on OR team members and patient safety ${ }^{1,4-6}$. Nevertheless, the literature confirms distractions and interruptions are a leading stressor for the entire OR team, contributing to unfavourable clinical performance, jeopardising patient care and, potentially, resulting in patient harm ${ }^{1,3,4}$.

Distractions and interruptions impact communication and team coordination, increase workload and fatigue, disturb concentration and situational awareness and impact workflow ${ }^{3,4,7}$. This can result in errors, delays, increases in surgical duration and cost, and omission of safety checks ${ }^{1,4-8}$. It is therefore essential for distractions and interruptions to be minimised $d^{1,3-8}$.

Distractions and interruptions are defined slightly differently between authors. Generally distractions are events which potentially divert one's attention from the primary task and interruptions occur when distractions are responded to, rapidly interrupting and switching attention away from the primary task $1,4,6,7,9,10$. Psychology and neuroscience research shows shifting attention from a primary task to a secondary task can be detrimental as it increases cognitive load and forces one to perform a dual task, or multi-task ${ }^{1,2,0,11}$.

This integrative review explores the impact of distractions and interruptions in the OR on patient safety and the OR team. Despite the paucity and heterogeneity of the literature, the various types, frequency, severity and impacts of distractions and interruptions in real OR settings will be presented, in addition to controlled experiments in simulation laboratories studying the impacts of specific distractions and interruptions.

\section{Literature search}

An integrative review methodology was used in this review as outlined by Whittemore and Knafl ${ }^{12}$. This method allows varied methodologies including qualitative and quantitative to be included to assist in presenting an extensive and holistic view of a phenomenon ${ }^{12}$. An electronic search was conducted to identify suitable literature. Cumulative Index of Nursing and Allied Health Literature (CINAHL) Complete, Medline Complete, PubMed, Scopus, Joanna Briggs Institute EBP and Cochrane Library databases were searched. The reference lists of selected papers were also searched. The search terms, truncations and Boolean operators used were 'distract* OR interrupt* OR disrupt*' AND 'operating room OR operating theatre OR perioperative' AND 'patient safety'.

\section{Inclusion and exclusion criteria}

Limiters on database searches were applied, including publication years 2014 to 2019, English language, peerreviewed (in CINAHL Complete), and full-text. The timeframe was applied to ensure the most contemporary papers were identified. Further inclusion criteria included primary research papers using quantitative, qualitative or mixed-methods methodology; primary outcomes of distraction, interruption or disruption; and settings within an OR or a simulation laboratory. Exclusion criteria included non-primary research, quality improvement studies, reviews, opinion pieces, guidelines, observational studies focused on only one distraction, and primary research where patient safety was not a focus.

\section{Data evaluation synthesis}

\section{Data extraction}

The titles and abstracts from qualitative, quantitative and mixedmethods papers were reviewed against the inclusion and exclusion criteria. Data extracted included lead author, published year, country, aim, design, sample, key findings and study limitations.

\section{Data evaluation}

The included papers were critically assessed for methodological quality with the mixed methods appraisal tool (MMAT). This tool covers five categories of study design including qualitative, quantitative non-randomised, descriptive or randomised control trials, and mixedmethods ${ }^{13-15}$. The efficiency, validity and reliability of the MMAT tool are well supported ${ }^{13,14}$. Each category incorporates criteria questions which can be answered and thereby scored between zero and two; 'no' (zero), 'cannot tell' (one) and 'yes' (two). These scores were converted into percentages. The critical appraisal skills programme (CASP) tools were also used to confirm quality ${ }^{16,17}$.

\section{Data synthesis}

As per Whittemore and Knafl' ${ }^{12}$ the included studies were synthesised using thematic analysis to distinguish themes, differences and relationships. Two categories of studies were determined, observational in ORs and experimental in simulation 
laboratories. Themes identified under the category of observational studies include types, frequency and severity of distractions and interruptions, and impacts on patient safety and the OR team. Under the simulation experimental category, themes identified included types of distractions and interruptions, and impact on mental workload, clinical decision-making, surgical performance and nurses.
Table 1: Search results

\begin{tabular}{|l|c|}
\hline Database & $\begin{array}{c}\text { Number } \\
\text { of articles }\end{array}$ \\
\hline PubMed & 120 \\
\hline Medline Complete & 48 \\
\hline CINAHL Complete & 64 \\
\hline Scopus & 28 \\
\hline Cochrane Library & 26 \\
\hline JBI & 10 \\
\hline From references & 1 \\
\hline
\end{tabular}

\section{Findings}

\section{Descriptive findings}

The database search identified 296 articles from six databases and one study was found through searching reference lists (see Table 1). Duplicates were removed, leaving 195 titles and abstracts which were screened against the exclusion criteria. Sixteen full-text studies were reviewed; however, two were excluded as the primary measures were not distractions or interruptions. As shown in Figure 1, the preferred reporting items for systematic reviews and meta-analyses (PRISMA) flow diagram, 14 studies were

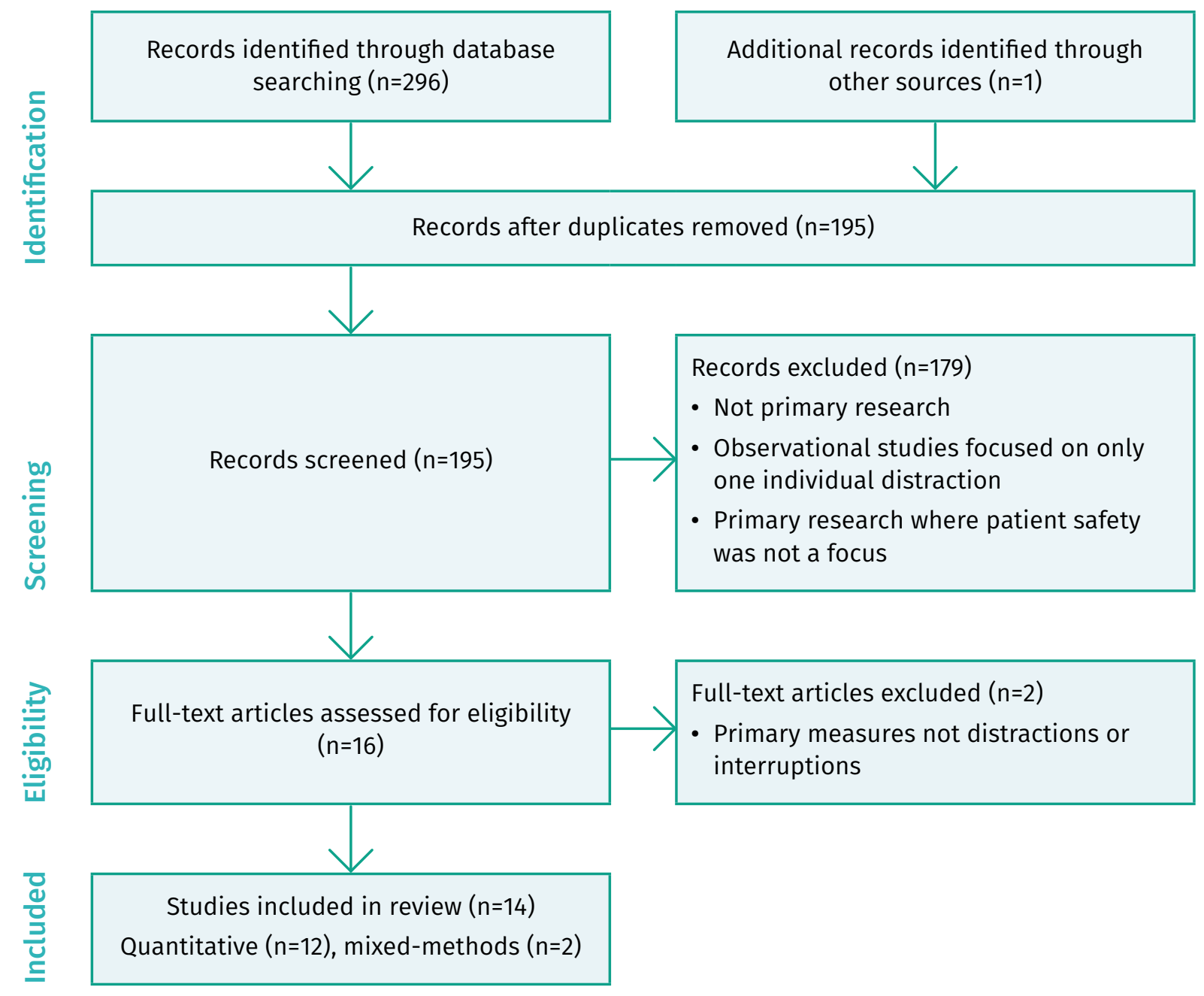

Figure 1: PRISMA flow diagram of papers for inclusion (Moher et al. ${ }^{18}$ ) 
included in the review ${ }^{18}$. Twelve of these were quantitative studies and two were mixed-methods. Four studies were conducted in Germany, four in the United Kingdom, two in the United States of America, two in Canada, one in China and one across Australia, Thailand and China. The key descriptors of each included study are presented in Table 2 (see supplemental documents). These include primary author, published year, country, design and sampling, study aim, key findings, limitations, implications and MMAT score.

\section{Quality assessment}

The methodological quality MMAT scores of the 14 papers ranged from 60 to 90 per cent. The seven quantitative observational studies all scored 60 per cent, while the quantitative simulation experimental studies scored between 60 to 90 per cent, averaging 76 per cent. The two mixed-methods papers both scored 83 per cent against the mixedmethods criteria.

\section{Discussion of findings}

Observational studies conducted in real ORs and simulation studies performed in mock OR simulation laboratories are complementary and are able to present diverse data, adding to the knowledge and evidence of distractions and interruptions ${ }^{5}$. However, both types have limitations as well as advantages. Observational studies are inherently subjective, whereas simulation studies are controlled and able to assess objective outcomes, inferring causality, but are not conducted in real ORs ${ }^{5,8}$. Observational studies use validated and reliable measuring tools to provide a thorough investigation of distractions and interruptions in ORs'. Simulation studies have the advantage of studying clinicians performing a primary task while adding a secondary task; this would be unethical and unsafe to conduct with real patients ${ }^{2,19}$. Virtual reality simulators are validated to measure surgical performance; however, generalisability of simulated studies to real ORs is limited ${ }^{19}$. This integrated literature review has identified themes associated with both types of settings to provide a thorough overview of distractions and interruptions in the OR.

\section{Observational studies}

Seven quantitative observational studies were included in this review ${ }^{1,3,5,-9,11}$. All had small samples, frequently from a single hospital and covering limited specialties yet

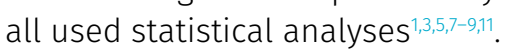
Two mixed-methods studies also incorporated quantitative observational study components within their studies 6,20 .

\section{Types, frequency and severity of distractions and interruptions}

\section{Types}

Various distractions and interruptions are discussed in the nine studies; however their heterogeneity is apparent as each study categorised types of distractions differently 1,3,5-9,11,20. Types include traffic (personnel entering and exiting the OR), phones/pagers, radio, case-irrelevant communications (CIC - i.e. communication not regarding the patient in the $O R$ ), teaching, movement (in front of monitors), crying babies (in caesarean cases), equipment, environmental, procedural, patient, and co-ordination issues, 1,3,-9,11,20. Four studies used the same tool which was developed by Healey et al. ${ }^{21}$ in 2006; however, each study modified it to develop different categories. The number of categories in each study ranged from five to twelve, illustrating the types of distractions and interruptions experienced by OR teams is significant 1,3,5-9,1,20. $^{\text {. }}$.

\section{Frequency}

Amongst the studies conducted in ORs, seven focus on distractions and interruptions affecting the whole OR team ${ }^{1,3,5,7-9,11}$, one on anaesthetists in the preoperative period $^{20}$ and another on nurses ${ }^{6}$. Due to the heterogeneity of the literature, it is difficult to determine the overall frequency of each type of distraction and interruption. Seven studies present the number per hour, ranging from 3.6 to 21.7 per hour $1,5-9,11$, averaging out to 10.1 distractions or interruptions each hour, or significantly one every six minutes. The remaining two studies reported frequencies per patient; Al-Hakim et al. ${ }^{20}$ found three per patient in the preoperative period, and Jung et al. ${ }^{3}$ two per patient intraoperatively. Noting the heterogeneity of the studies, the highest frequency was $\mathrm{CIC}$, followed by phone/pager, equipment issues and traffic 1,3,5-9,11,20.

\section{Severity}

Of the nine observational studies, four used the tool developed by Healey et al. ${ }^{21}$ to measure types and severity of distractions and interruptions ${ }^{1,5,8,9}$. The validated tool for use in ORs uses a ninepoint nominal scale and measures visible severity relating to the OR team's involvement in an event $\mathrm{t}^{1,5,8,9}$. Scores between 1 and 3 indicate a distraction has potentially or actually affected the circulating nurse, between 4 and 6 suggests one other team member (excluding the circulating nurse) is distracted or interrupted, 7 or 8 means more than one member is affected, and 9 indicates surgical flow is impacted ${ }^{1,5,8,9}$.

These four studies trained two to three observers and measured high inter-rater agreement/reliability (IRR) 
during pilot studies ${ }^{1,5,8,9}$. In addition, two blinded the observers ${ }^{5,8}$. Importantly, the pilot period reduced the potential for the Hawthorne effect, whereby subjects alter their behaviour while being observed ${ }^{5}$. Despite this, the studies occurred in single hospitals and across minimal specialties, creating the possibility of selection bias ${ }^{1,5,8,9}$. Observer fatigue and observer bias are also possibilities ${ }^{1,5,8,9}$. In addition, Sevdalis et al. ${ }^{5}$ observed a single surgeon's procedures. Despite using the same tool, discrepancies exist regarding which distractions and interruptions had the highest severity ${ }^{1,5,8,9}$. Acknowledging the heterogeneity, equipment issues had the highest severity followed by procedural issues and $\mathrm{CIC}^{1,5,8,9}$.

Two further studies used another validated tool comprising three levels $s^{7,1}$. Level 1 events are dealt with by the unscrubbed team members (not in the sterile field, for example circulating nurse and anaesthetists), 711 . Level 2 affects one member of the scrubbed staff (within the sterile field) and level 3 affects more than one member, including the primary surgeon ${ }^{7,11}$. Interestingly both studies showed the same three highest severity distractions or interruptions (level 2 or 3), however in different orders: CIC, others, equipment ${ }^{7}$, compared to equipment, $\mathrm{CIC}$ and others ${ }^{11}$. Notably, Yoong et al. ${ }^{11}$ determined the three most frequent were also the three most severe. Over 11 per cent of total operative time involved a level 2 or 3 distraction in Willett et al.'s ${ }^{7}$ study. Both studies triangulated the data and used independent observers; Willett et al. ${ }^{7}$ did not report on the training of observers, Yoong et al. ${ }^{11}$ did, and neither reported on IRR.

The remaining studies used different measures. Jung et al. ${ }^{3}$ measured post-operatively using a humanfactors, self-reported questionnaire (surgical team assessment record or STAR) to measure the primary surgeon's perceived distraction ${ }^{3}$. Findings were that OR doors opening and $\mathrm{CIC}$ had the highest severity ${ }^{3}$. Significantly, this tool is subjective; the validity of it has not been completely established and only a single surgeon was studied limiting the generalisability despite a large sample of $265^{3}$.

Severity was also measured postoperatively in Al-Hakim et al.'s ${ }^{20}$ mixed-method study; however, only anaesthetists were in the study group and severity was measured by the amount of time wasted. Times were analysed against semi-structured interview responses regarding perceptions of care coordination issues. The authors established distractions and interruptions caused by staff and coordination within the OR team had the largest impact ${ }^{20}$. This study occurred in five hospitals across three countries; however, the observers and interviews have the potential to be biased due to subjectivity ${ }^{20}$. Sirihorachai et al.'s. ${ }^{6}$ mixed-methods study is the only one conducted by nurses, and studied only nurses. A validated tool comprising four levels was used: for level 1 the circulating nurse does not respond, for level 2 the primary task is ceased and the secondary task attended to, for level 3 the nurse multitasks, and for level 4 the operation flow is interrupted ${ }^{6}$. The highest severity distraction or interruption was CIC followed by equipment issues and phone/music/ pager $^{6}$. The potential for observer bias existed here due to subjectivity and the use of one observer and therefore no IRR ${ }^{6}$. In addition, a single centre and specialty allows for possible selection bias ${ }^{6}$.

Inconsistent categories and tools make it is impossible to ascertain which distractions and interruptions have the highest severity. However, equipment issues comparatively appear to have the highest severity followed by $\mathrm{CIC}$ and procedural issues $1,3,5-9,1,20$. Therefore, the frequency and severity of distractions and interruptions are not correlated ${ }^{1}$.

Impacts of distractions and interruptions

\section{Mental workload}

Understanding and studying the impact distractions and interruptions have on mental workload is crucial to understanding stress, burnout, training requirements, OR team needs and system demands ${ }^{4}$. Three observational studies used mental workload measurements to determine the association between the frequency and severity of distractions and interruptions and the perceived mental workload of the OR team ${ }^{3,8,9}$. Weber et al. ${ }^{9}$ and Weigl et al. ${ }^{8}$ used the validated surgery task load index (SURG-TLX) questionnaire which enables subjective assessments, differentiates between complexities of tasks, and specifies objective performance. The OR team answered questions using three elements of the tool: mental demands, situational stress, and distraction ${ }^{8,9}$. Weber et al. ${ }^{9}$ added productivity and perceived quality. The subjectivity of the tool allows for potential subjectivity and recall bias $^{8,9}$.

According to Weigl et al. ${ }^{8}$ the perceived mental workload for all team members is correlated to severity of distractions and interruptions. Results were different for each profession. For surgeons, $\mathrm{CIC}$ was linked to a decrease in situational stress, yet an increase in perceived distraction; however, individual surgeons respond differently to individual types ${ }^{8}$. This indicates some CIC and small talk may be positive and reduce surgeons' fatigue and stress ${ }^{8}$. Nurses' 
situational stress was negatively correlated with telephone/pager calls which were the most frequent and severe, and anaesthetists found $\mathrm{CIC}$ the most distracting ${ }^{\text {. }}$. Overall, reducing $\mathrm{CIC}$ and phone/ pager distractions and interruptions reduces the risk of a cumulative effect upon mental workload ${ }^{8}$.

Mental workload and the severity of distractions and interruptions due to coordination and communication revealed statistically significant correlation in Weber et al.'s study using Pearson correlation ( $p=<$ $0.05)^{9}$. Interestingly, post-operative reporting using the SURG-TLX tool showed anaesthetists $(n=42)$ reported higher levels of mental demands than surgeons $(n=81)$ and nurses $(n=93)^{9}$. Anaesthetists and nurses reported higher distraction rates than surgeons and $\mathrm{CIC}$ was linked to higher stress in anaesthetists although this study only observed robotic prostatectomies in a single hospital? The SURG-TLX assesses perceived workload post-operatively; it does not consider workload at different time points intra-operatively.

The study by Jung et al. ${ }^{3}$ used the human-factors STAR tool and, in contrast to the previous studies, did not include anaesthetists or nurses but just a single surgeon. Through a multivariable analysis, $\mathrm{CIC}$ was independently correlated with an increase in surgeon's distraction ${ }^{3}$, a similar finding to Weigl et al. ${ }^{8}$. $\mathrm{CIC}$ is a modifiable distraction and interruption which appears to affect team members' mental workload differently ${ }^{3}$. Weber et al. ${ }^{9}$ state $\mathrm{CIC}$ decreases mental fatigue and stress, yet Weigl et al. ${ }^{8}$ found this was not the case for all OR professions, in particular anaesthetists. However, all three authors agree $\mathrm{CIC}$ should be minimised in the OR to reduce the mental workload of all staff $3,8,9$.

\section{Patient outcomes and safety}

Unlike simulation studies, observational studies are unable to infer direct causality, yet four of the nine observed and recorded patient safety and outcome variables 5,71,20. Yoong et al.1 and Willett et al. ${ }^{7}$ found no post-operative complications or adverse events occurred in any patient, despite distractions and interruptions being prevalent. Both measured case prolongation due to distractions and interruptions - 18.45 minutes per case ${ }^{11}$ and 11.05 minutes per case respectively ${ }^{7}$. Similarly, AlHakim et al..$^{20}$ determined distractions and interruptions added just under a minute to each preoperative period. This has the potential to increase the risk of adverse patient outcomes and cost and decrease efficiency?. Sevdalis et al. ${ }^{5}$ identified distractions involving communication were related to lower completion of patient safety checks. More distractions led to a decline in the number of intra-operative checks completed $^{5}$. Alarmingly, the teams were experienced, yet endangered patient safety by not completing checks ${ }^{5}$. However, this study was conducted in a single hospital with a single surgeon so generalisability and selection bias is questionable ${ }^{5}$.

\section{OR team members}

Distractions and interruptions affect different OR professions differently 1,5,8, . Antoniadis et al. found circulating and anaesthetic nurses were impacted more by the highly prevalent distractions and interruptions caused by traffic and phone calls/pagers as they attended to them while attempting to perform their primary tasks. Similarly, Sirihorachai et al. ${ }^{6}$ found nurses were most distracted by traffic and phones/pagers occurring during critical times of induction, counting and specimen handling. Nurses protected surgeons from traffic and phones by refraining from asking questions or passing on information at critical times during procedures ${ }^{5}$. Traffic and phone calls can be minimised and nurses are able to develop policies and guidelines to ensure this occurs ${ }^{1,5,6}$.

There is paucity in the recent observational literature analysing the effect of distractions and interruptions on anaesthetists and nurses with the majority of studies focussing on surgeons. The heterogeneity makes it is impossible to ascertain which distractions have the largest impact. Regardless, all nine studies agree that unnecessary distractions should be minimised, and those which cause the whole team to be distracted or interrupted have the largest impact $1,3,5-9,11,20$.

\section{Simulation studies}

Five simulation studies have been included in this review ${ }^{2,4,10,19,22}$, and one mixed-methods study by Sirihorachai et al. ${ }^{6}$ which combined observational and simulation components. There is also paucity in the simulation literature studying anaesthetists and nurses - no anaesthetic studies were found and Sirihorachai et al. ${ }^{6}$ was the sole nursing study. The five surgeon studies all applied a distraction to novice subjects (medical students or surgical trainees) while they were performing a surgical technique or procedure on a simulator. Clinical decision-making and surgical performance were measured 2,4,10,19,22.

\section{Type of distractions and interruptions applied}

To improve generalisability of simulation studies, realistic OR distractions should be applied as secondary tasks while subjects are performing primary tasks ${ }^{19}$. Four studies applied an auditory and cognitive distraction including phones, pagers and patient complaints of pain 4,10,19,22. However, 
Gao et al. ${ }^{2}$ used mental arithmetic. Sirihorachai et al. ${ }^{6}$ applied a variety of distractions to nurses. Weigl et al. ${ }^{4}$ randomised 19 junior surgeons to one of two groups; the first were distracted by a phone call, the second by patient discomfort, while performing a vertebroplasty. In the phone call group, the caller insisted on speaking to the surgeon and in the other group, the simulated patient complained of pain, requiring the surgeon to administer more local anaesthetic ${ }^{4}$. Sujka et al. ${ }^{22}$ studied 12 residents each performing six laparoscopic cholecystectomies, three with pager distractions based on clinical questions regarding ward patients and three with no distraction. The order these were performed was randomised 22 .

Similarly, Murji et al. ${ }^{19}$ used pager distractions and asked questions regarding a pre-read handover sheet. Thirty residents performed laparoscopic salpingectomies either distracted or undistracted, in randomised order ${ }^{19}$. Yang et al.10 used mild and strong phone call distractions involving clinical questions. Thirty medical students were distracted mildly, strongly or not at all, while they performed an easy and difficult laparoscopic task $^{10}$. Sirihorachai et al. ${ }^{6}$ applied seven distractions at critical times, including the first and final counts, and team time out to 30 nurses. Distractions included $\mathrm{CIC}$, pager, music, extra equipment and dropping of an instrument.

In contrast, Gao et al. ${ }^{2}$ applied arithmetic questions to 24 medical students. The students answered without operating, and performed a laparoscopic appendicectomy with the arithmetic and without; the order was randomised ${ }^{2}$. The authors believe arithmetic is a cognitive task and therefore appropriate to use as a secondary task. However, Murji et al..$^{19}$ disagree, stating arithmetic is not a meaningful or realistic secondary task.

\section{Impacts of distractions and interruptions}

\section{Mental workload}

Three studies measured the outcome of mental workload using different tools, adding further to the heterogeneity ${ }^{2,4,10}$. Weigl et al. used the SURG-TLX and determined surgeons' perceived workload was statistically significantly higher when distracted than when not distracted $(p<0.01)$. The subjects also experienced increased physical demands and situational stress 4 . Interestingly, mental workload was statistically significant when associated with surgical inaccuracy $(p=0.04)^{4}$. However, this tool is subjective, only measures workload post-operatively and was only used on junior surgeons ${ }^{4}$. Similarly, Gao et al. ${ }^{2}$ used the National Aeronautics and Space Administration (NASA) task load index (NASA-TLX), which the SURG-TLX is adapted from ${ }^{8}$. The authors also used an objective measure to track pupil size and blink rate which represent cognitive load ${ }^{2}$. When performing the dual-task of answering arithmetic questions and operating, mental workload and eye measurements of medical students were higher than those measured during the single task of operating ${ }^{2}$. It is unclear why the SURG-TLX was not used as it is specific to surgery; in addition, the use of arithmetic is questionable 8,19 .

Yang et al.10 did not report on their tool; it is assumed subjects rated their distraction levels postoperatively. Subjects reported being more affected when a strong distraction was applied $(p<0.05)^{10}$. No return rate or validity information was reported and only medical students were studied ${ }^{10}$. As in the observational studies, it is apparent that when distracted or interrupted novice surgeons experience a higher mental workload than when they are not.

\section{Surgical performance}

Surgical performance was measured on simulators; each study used different outcomes including inaccuracy, time to complete, safety, complications, blood loss and specific surgical markers 2,4,10,19,22. As discussed, Weigl et al. ${ }^{4}$ found a statistically significant correlation between increased mental workload and inaccuracy ( $p=0.04)$. Similarly, Yang et al.'s ${ }^{10}$ results showed when performing an easy and hard task while distracted, accuracy decreased. Gao et al. ${ }^{2}$ found several surgical performance factors were diminished when subjects were distracted. The time taken to complete the task was not affected by distraction in three studies 4,10,22; however, it was prolonged in two studies ${ }^{2,19}$. No difference was determined in blood loss, complications or safety between distracted and non-distracted surgery ${ }^{19,22}$. Studies demonstrated surgical performance is diminished and more inaccuracies occur when novice surgeons are distracted or interrupted ${ }^{2,4,10,19,22}$. However, the studies have small, selective samples and do not include experienced surgeons ${ }^{2,4,10,19,22}$.

\section{Clinical decision-making}

Three studies measured clinical decision-making as an outcome, two with pager distractions and one with phone calls ${ }^{10,19,22}$. These clinical decisions regarded invented ward patients - in ORs, surgeons operate (primary task) while answering clinical questions regarding other patients (secondary task) $)^{10,19,22}$. Sujka et al. ${ }^{22}$ established that, when distracted, surgical residents correctly resolved clinical issues only 25 per cent of the time. 
This may be due to the residents focusing on the surgical task, rather than the secondary task, as they were blinded to the purpose of the study ${ }^{22}$. Likewise, 63 per cent of residents in a powered sample made a minimum of one unsafe clinical decision when distracted with questions; the mean for correct answers was 80 per cent ${ }^{19}$. Similarly, medical students made more errors when answering questions from two phone calls in the final study ${ }^{10}$. This raises the question about patient safety and care of ward patients managed by surgeons while they are operating $10,19,22$. However, these studies did not evaluate the effect on experienced surgeons ${ }^{10,19,22}$.

\section{Nurses}

In the simulation laboratory, nurses were distracted while performing first and final counts and team time out ${ }^{6}$. Measures included whether the nurses ignored the distraction, were interrupted by it, or multitasked and performed both the primary and secondary task ${ }^{6}$. Interestingly, all the more experienced nurses (greater than two years) performed team time out with the radio on despite it breaching policy while all the junior nurses turned it off ${ }^{6}$. Over half of the nurses were interrupted by $\mathrm{CIC}$ and pager distractions during the first count ${ }^{6}$. Multitasking only occurred during the first count; ten per cent of nurses engaged in $\mathrm{CIC}$ while counting ${ }^{6}$. A third of nurses were interrupted by a pager during team time out and 57 per cent at the final count ${ }^{6}$. This is concerning as these three tasks are critical to ensure patient safety ${ }^{6}$. That said the 30 nurses came from a single centre, making generalisability difficult and selection bias possible ${ }^{6}$. Debriefing sessions and qualitative analysis revealed the nurses used cognitive processes of prioritisation and remaining focused on the primary task when confronted with distractions ${ }^{6}$.

\section{Implications for perioperative nursing practice or research}

This review has highlighted reducing distractions and interruptions is essential to enhance patient safety and productivity; maintain safe and effective care, performance, workload and communication; and decrease and mitigate the potential risk to the OR team ${ }^{1,3,8,11}$. Multidisciplinary collaboration and system-level strategies are required ${ }^{1,3}$. Improvements in multidisciplinary communication, information transfer, organisation and collaboration are essential for smooth surgical flow ${ }^{1,6,8,9}$. Distractions and interruptions are usually an indication of system issues which are often upstream from the OR and lead to a lack of coordination between the OR and other departments; therefore, thorough system analyses and improvements are required 5,9,20.

Education and training are the initial approaches for resolving system and multidisciplinary coordination issues ${ }^{6,79}$. Multidisciplinary education should create an awareness of the different types of distractions and interruptions which occur in the OR and focus on the potential severity and impact of each ${ }^{6}$. Each profession is impacted differently by individual types and a clear understanding of this from the entire OR team will assist in minimising them ${ }^{6-9}$. Education may include simulations to further enhance awareness between professions and assist in developing effective strategies ${ }^{6,9}$. Nurses should be taught how to prioritise and stay focused on primary tasks, especially during critical phases ${ }^{6}$.

Nurses can influence policy and conduct ongoing quality improvement projects in their own ORs to minimise distractions and interruptions ${ }^{5,6}$. Quality improvement projects should include observing staff over time to assess frequency and severity of distractions and interruptions ${ }^{5}$. Feedback should be provided to the OR team, followed by discussion to identify effective actions and strategies ${ }^{5}$. Reassessment should occur post implementation ${ }^{5}$. Nursing professional bodies should develop standards and guidelines for minimising distractions and interruptions in $\mathrm{ORs}^{6}$.

Several effective strategies have been implemented in numerous ORs $\mathrm{OR}^{3,5,8,11}$. The sterile cockpit is an aviation concept successfully adapted to the OR environment ${ }^{3,5,8}$. This involves eliminating non-essential communication during critical phases of a procedure in order to enhance patient safety and reduce effects on the OR team ${ }^{5,8}$. Preoperative briefings enable effective planning and organisation, reducing unnecessary distractions and interruptions s,7,11. $^{5}$.

Further research is essential to fully understand the phenomenon of distractions and interruptions in the OR. Research determining the cumulative effects of avoidable distractions and interruptions on the OR team is required ${ }^{1,4,9}$. Additional suggestions for research include complex and emergency surgery, OR team familiarity, individuals' stress management strategies and ascertaining the ideal work process design ${ }^{5,8,9}$. Robust research is necessary to clearly determine which distractions and interruptions have the largest impact on mental workload and lead to adverse patient outcomes and unsafe practice ${ }^{1,4,8}$. Researching $\mathrm{CIC}$ to clearly delineate between positive and negative $\mathrm{CIC}$ is vital ${ }^{1,8}$. Robust studies involving experienced professionals would resolve the paucity in the literature ${ }^{3,4,10,19}$. It is suggested 
that greater experience enables a member of the OR team to develop compensatory measures, resilience and strategies which increase their immunity to the impact of distractions and interruptions ${ }^{3,4,10,19}$.

\section{Limitations}

This review has several limitations. Despite a thorough and systematic search, some papers may have unintentionally been omitted. Papers not written in English were excluded but may have included important research. No qualitative studies were found, yet such research would have enhanced a holistic review of the phenomenon. The quality of the included studies was assessed by one individual and despite using two validated tools (MMAT and (ASP), subjectivity was not able to be controlled. Paucity in the recent literature of nursing and anaesthetic studies, particularly in the simulation studies, directed the review to a stronger focus on surgeons and surgical technique. However, this remains vital when discussing the impact on the entire OR team. Overall the heterogeneity of the literature limited consensus regarding which distractions and interruptions have the largest impact on each profession and patient safety.

\section{Conclusion}

This integrative review has provided a thorough overview of the recent literature on distractions and interruptions in the OR. It is of concern these studies confirm a distraction or interruption occurs on average every six minutes. It is evident that patients and the OR team are impacted significantly, yet through system analyses, education, planning, research and local quality improvement projects many of these impacts can be avoided. Nurses are central to improving and creating positive change in the perioperative environment. With guidance from professional OR nursing bodies, nurses can develop and implement standards and local policies to reduce the frequency, severity and impact of distractions and interruptions upon their patients, colleagues and OR teams.

\section{Acknowledgement}

This paper was submitted to the University of Tasmania for assessment in the subject CNA803 as part fulfilment of Advanced Clinical Nursing Practice for Master of Clinical Nursing (Perioperative Nursing). The author sincerely wishes to thank $\mathrm{Dr}$ Paula Foran, unit coordinator, for her guidance throughout the master course and encouragement to finalise and submit this paper.

\section{References}

1. Antoniadis S, Passauer-Baierl S, Baschnegger H, Weigl M. Identification and interference of intraoperative distractions and interruptions in operating rooms. J Surg Res 2014;188(1):21-9. DOI: 10.1016/j. jss.2013.12.002.

2. Gao J, Liu S, Feng Q, Zhang X, Jiang M, Wang $L$ et al. Subjective and objective quantification of the effect of distraction on physician's workload and performance during simulated laparoscopic surgery. Med Sci Monit 2019;25:3127-3132. DOI: 10.12659/ msm.914635.

3. Jung JJ, Elfassy J, Grantcharov T. Factors associated with surgeon's perception of distraction in the operating room. Surg Endosc 2019;34(7):3169-3175. DOI: 10.1007/ s00464-019-07088-z.

4. Weigl M, Stefan P, Abhari K, Wucherer P, Fallavollita P, Lazarovici M et al. Intraoperative disruptions, surgeon's mental workload, and technical performance in a full-scale simulated procedure. Surg Endosc 2016;30(2):559-566. DOI: 10.1007/s00464-0154239-1.

5. Sevdalis N, Undre S, McDermott J, Giddie J, Diner L, Smith G. Impact of intraoperative distractions on patient safety: A prospective descriptive study using validated instruments. World J Surg 2014;38(4):751-758 DOI: 10.1007/s00268-013-2315-z.
6. Sirihorachai R, Aebersold ML, Sarter NB, Harris M, Marsh V, Redman RW. Examining interruptions in the operating room using simulation. Clinical Simulation in Nursing 2018;24:14-24. DOI: 10.1016/j.ecns.2018.08.004.

7. Willett M, Gillman O, Shin E, Sewart E, Muller $D$, Nauta $M$ et al. The impact of distractions and interruptions during cesarean sections: A prospective study in a London teaching hospital. Arch Gynecol Obstet 2018;298(2):313-318. DOI: 10.1007/s00404-0184810-9.

8. Weigl M, Antoniadis S, Chiapponi C, Bruns C, Sevdalis N. The impact of intra-operative interruptions on surgeons' perceived workload: An observational study in elective general and orthopedic surgery. Surg Endosc 2015;29(1):145-153. DOI: 10.1007/ s00464-014-3668-6.

9. Weber J, Catchpole K, Becker AJ, Schlenker $B$, Weigl M. Effects of flow disruptions on mental workload and surgical performance in robotic-assisted surgery. World J Surg 2018;42(11):3599-3607. DOI: 10.1007/s00268018-4689-4

10. Yang C, Heinze J, Helmert J, Weitz J, Reissfelder C, Mees ST. Impaired laparoscopic performance of novice surgeons due to phone call distraction: A single-centre, prospective study. Surg Endosc 2017;31(12):5312-5317. DOI: 10.1007/ s00464-017-5609-7.

11. Yoong W, Khin A, Ramlal N, Loabile B, Forman S. Interruptions and distractions in the gynaecological operating theatre: Irritating or dangerous? Ergonomics 2015;58(8):1314-1319. DOI: 10.1080/00140139.2015.1005171.

12. Whittemore $R$, Knafl $K$. The integrative review: Updated methodology. J Adv Nurs 2005;52(5):546-553. DOI: 10.1111/j.13652648.2005.03621.x

13. Pluye $\mathrm{P}$, Hong QN. Combining the power of stories and the power of numbers: Mixed methods research and mixed studies reviews. Annu Rev Public Health 2014: 35:29-45. DOI: 10.1146/annurevpublhealth-032013-182440.

14. Souto R, Khanassov V, Quan Nha H, Bush PL, Vedel I, Pluye P. Systematic mixed studies reviews: Updating results on the reliability and efficiency of the mixed methods appraisal tool. Int J Nurs Stud 2015;52(1):500501. DOI: 10.1016/j.ijnurstu.2014.08.010.

15. Pace R, Pluye P, Bartlett G, Macaulay AC, Salsberg J, Jagosh J et al. Testing the reliability and efficiency of the pilot mixed methods appraisal tool (MMAT) for systematic mixed studies review. Int J Nurs Stud. 2012;49(1):47-53. DOI: 10.1016/j. ijnurstu.2011.07.002. 
16. Critical Appraisal Skills Program (CASP). CASP checklist: 12 questions to help you make sense of a cohort study [Internet]. Oxford: CASP; 2018 [cited 2020 August 19]. Available from: casp-uk.net/casp-toolschecklists/.

17. Critical Appraisal Skills Program (CASP). CASP checklist: 11 questions to help you make sense of a randomised control trial [Internet]. Oxford: CASP; 2018 [cited 2020 August 19]. Available from: casp-uk.net/ casp-tools-checklists/.
18. Moher D, Liberati A, Tetzlaff J, Altman DG, Group P. Preferred reporting items for systematic reviews and meta-analyses: The PRISMA statement. PLoS Med 2009;6(7):e1000097. DOI: 10.1371/journal. pmed.1000097.

19. Murji A, Luketic L, Sobel ML, Kulasegaram KM, Leyland N, Posner G. Evaluating the effect of distractions in the operating room on clinical decision-making and patient safety. Surg Endosc 2016;30(10):4499-4504. DOI: 10.1007/s00464-016-4782-4.
20. Al-Hakim L, Arora S, Sevdalis N. Impact of disruptions on anaesthetic workflow during anaesthesia induction and patient positioning: A prospective study. Eur J Anaesthesiol 2016;33(8):581-587. DOI: 10.1097/ eja.0000000000000484.

21. Healey AN, Sevdalis N, Vincent CA. Measuring intra-operative interference from distraction and interruption observed in the operating theatre. Ergonomics 2006 ;49(56):589-604. DOI: 10.1080/00140130600568899.

22. Sujka JA, Safcsak K, Bhullar IS, Havron WS $3^{\text {rd }}$. Simulation-based testing of pager interruptions during laparoscopic cholecystectomy. J Surg Educ 2018;75(5):13511356. DOI: 10.1016/j.jsurg.2018.01.012. 